\title{
Uncertainty Analysis of the Grazing Flow Impedance Tube
}

\author{
Martha C. Brown, ${ }^{*}$ Michael G. Jones, ${ }^{\dagger}$ and Willie R. Watson ${ }^{\ddagger}$ \\ NASA Langley Research Center, Hampton, VA 23681-2199 USA
}

This paper outlines a methodology to identify the measurement uncertainty of NASA Langley's Grazing Flow Impedance Tube (GFIT) over its operating range, and to identify the parameters that most significantly contribute to the acoustic impedance prediction. Two acoustic liners are used for this study. The first is a single-layer, perforateover-honeycomb liner that is nonlinear with respect to sound pressure level. The second consists of a wire-mesh facesheet and a honeycomb core, and is linear with respect to sound pressure level. These liners allow for evaluation of the effects of measurement uncertainty on impedances educed with linear and nonlinear liners. In general, the measurement uncertainty is observed to be larger for the nonlinear liners, with the largest uncertainty occurring near anti-resonance. A sensitivity analysis of the aerodynamic parameters (Mach number, static temperature, and static pressure) used in the impedance eduction process is also conducted using a Monte-Carlo approach. This sensitivity analysis demonstrates that the impedance eduction process is virtually insensitive to each of these parameters.

$\begin{array}{ll}c & =\text { speed of sound } \\ C I_{\text {next }} & =95 \% \text { confidence interval of next measurement } \\ C I_{\text {mean }} & =95 \% \text { confidence interval of true mean } \\ d & =\text { liner facesheet hole diameter, in } \\ f & =\text { frequency, } \mathrm{Hz} \\ h & =\text { liner core cell (cavity) depth, in } \\ i & =\text { free space wavenumber, } \mathrm{ft}^{-1} \\ k & =\text { exit plane of the computational domain, in } \\ L & =\text { axial locations of leading and trailing edges of liner, in } \\ L_{1, L_{2}} & =\text { mean flow (uniform flow) Mach number } \\ M_{\text {ave }} & =\text { centerline Mach number } \\ M_{C / L} & =\text { number of trials } \\ N & =\text { number of microphones along wall } \\ n w a l l & =\text { complex acoustic pressure } \\ p & =\text { source and exit plane complex acoustic pressures } \\ p_{s}, p_{e} & =\text { flow resistance, lbm/lft }{ }^{2} \cdot \mathrm{s} \\ P_{s} & =\text { estimate of standard deviation for a small sample size }(\mathrm{N}<32) \\ R_{f} & =\text { sound pressure level, dB [ref } 20 \mu \text { Pa] } \\ S & =\text { liner facesheet thickness, in } \\ S P L & =\text { inverse of the Student's t-distribution } \\ t & =\text { static temperature, }{ }^{\circ} \mathrm{F} \\ t^{*} & =\text { three dimensional coordinate system, in } \\ T_{s} & \end{array}$

\footnotetext{
${ }^{*}$ Research Engineer, Aeroacoustics Branch, Liner Physics Group, MS 164D.

$\dagger$ Senior Research Scientist, Structural Acoustics Branch, Liner Physics Group, MS 463, Associate Fellow AIAA.

* Senior Research Scientist, Computational AeroSciences Branch, Liner Physics Group, MS 128, Associate Fellow AIAA.
} 


\section{Introduction}

$\mathrm{E}$ ngine fan noise is one of the key contributors to aircraft noise in the vicinity of airports. Although a number of enhancements have been introduced to reduce this noise source (e.g., sweep and lean of fan blades), acoustic liners mounted in the walls of the aircraft engine nacelle continue to provide a significant portion of fan noise reduction. The most pertinent parameter for understanding the acoustic performance of these liners is acoustic impedance, an intrinsic parameter that is dependent on sound pressure level and the normal component of acoustic velocity at the liner surface. Given the current trend toward higher-bypass ratio turbofan engines, with the corresponding reduction in liner effectiveness, there is a need for increased impedance model fidelity such that optimum liner impedances can be achieved more consistently.

For over 30 years, researchers at NASA Langley have concentrated on the development of multiple approaches to evaluate acoustic liners in realistic aeroacoustic environments. The NASA Langley Grazing Incidence Tube (GIT) was used for this purpose for over 20 years, and was recently replaced with the Grazing Flow Impedance Tube (GFIT) that is illustrated in Fig. 1. The GFIT provides enhanced capabilities over the GIT. These include the ability to operate in inlet and exhaust configurations by moving the source section from downstream to upstream of the test section, and to evaluate acoustic liners in the presence of different boundary layers by moving the test section along the length of the duct. The GFIT resides in the Liner Technology Facility (LTF), a state-of-the-art test facility whose purposes are: (1) to conduct fundamental research to quantify liner acoustic properties, and (2) to assess the acoustic performance of liners on a scale approaching that of a full-size engine. The GFIT is used by NASA Langley Research Center to evaluate acoustic liners in a controlled aeroacoustic environment, and to acquire data suitable for validation of impedance prediction models and aeroacoustic duct propagation codes. Clearly, the quality of the impedance eduction process is critically dependent on consistently high-fidelity aeroacoustic measurements. The objective of this paper is to provide a methodology for monitoring the fidelity of data acquired with the GFIT.

This paper outlines a methodology to identify the measurement uncertainty of the GFIT over its operating range and to identify the parameters that most significantly contribute to the acoustic impedance prediction. This investigation is an extension to the uncertainty analysis performed by Jones, et al. ${ }^{1}$ using GFIT's predecessor, the GIT, and is being conducted to: (1) identify measurement capabilities and limitations, (2) identify confidence limits for the measurement approach, (3) identify confidence limits for acoustic impedances predicted via the current NASA Langley impedance eduction methodology, and (4) provide guidance in planning future experiments. Sections II and III provide descriptions of the test facility and the data acquisition, and Section IV provides a description of the uncertainty metrics. Section V provides a description of the current experiment and Section VI provides a discussion of the acoustic impedance eduction process. Results and discussion are provided in Section VII, and concluding remarks are presented in Section VIII.

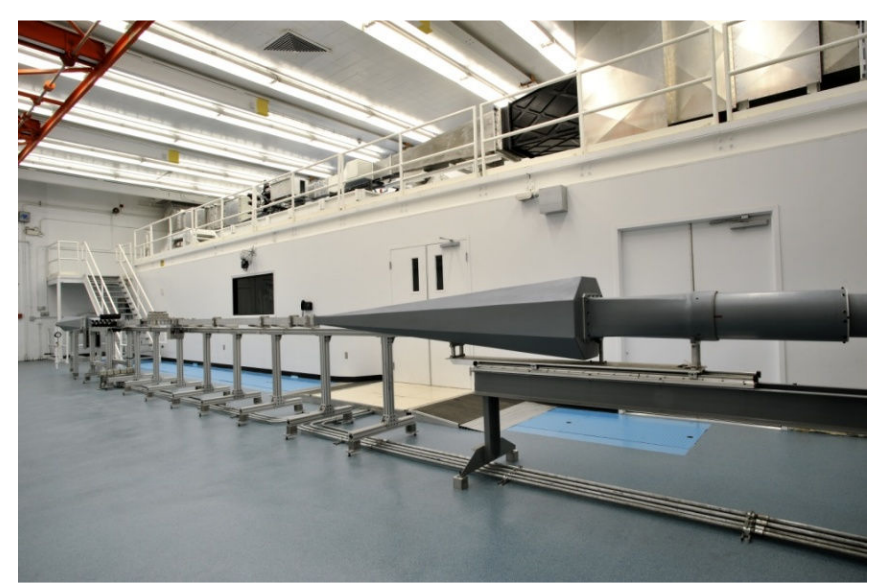

Figure 1. Photograph of Grazing Flow Impedance Tube (main floor) installed in NASA LaRC Liner Technology Facility (2009). 


\section{Description of Facility}

The Grazing Flow Impedance Tube is a waveguide used by NASA Langley Research Center to evaluate acoustic liners. The direction of flow is from left to right in Fig. 2, and the waveguide is configured in the exhaust configuration (sound and flow travelling in the same direction). Fig. 3 is a photograph depicting a close up view of the GFIT test section with microphone array. The components include an upstream near-anechoic settling chamber, a source section (also known as driver section) consisting of eighteen $120-\mathrm{W}$ acoustic drivers, the test section, a probe traverse section, and a downstream near-anechoic termination section. The GFIT consists of modular components that can be easily interchanged. This allows the test section to be positioned at multiple axial locations such that acoustic liners can be evaluated in different boundary layer regimes (fully laminar to fully turbulent). By interchanging the test section and the source section, the GFIT can also be configured simulate inlet conditions. Specific hardware described in this paper does not imply a recommendation or endorsement of any product or vendor.

Table 1 provides a synopsis of GFIT's characteristics and capabilities. The flow path consists of a test section of 2.0 by 2.5 inch cross-section, and acoustic liners of up to 24 inches in length can be evaluated. This cross-sectional geometry was chosen to ensure that higher-order modes in the horizontal and vertical dimensions cut on at different frequencies, thereby allowing their effects to be easily distinguished. The test section is populated with 95 Brüel and Kjær Type 4938 flush-mounted microphones that measure the complex acoustic pressures upstream, along the axial length, and downstream of the acoustic treatment. For the current investigation, the 53 microphones mounted in the wall opposite the liner are used for the analysis. The density of the microphone locations provides sufficient fidelity for educing the impedance at frequencies over the range of interest $(0.4$ to $3.0 \mathrm{kHz})$. The surface of the liner sample serves as the upper wall of the test section, with the capacity to test liner samples up to 3 inches deep.

The waveguide can be operated at mean flow velocities up to Mach 0.6 under ambient conditions. This is achieved by balancing the pressure in the test section from heated, high pressure air upstream of the test rig and a vacuum blower located downstream of the test rig. The high-pressure air entering the waveguide is heated in a controlled manner to achieve an adiabatic wall condition at the test section (as assumed in the duct propagation model described in Section VI). Sound generated with 18 acoustic drivers allows sound pressure levels up to 160 $\mathrm{dB}$ to be achieved over a frequency range of 0.4 to $3.0 \mathrm{kHz}$. The termination consists of a near-anechoic diffuser. Finally, for the current investigation, the GFIT is configured in the exhaust configuration, and the test section is positioned at the farthest possible downstream location to achieve a fully developed boundary layer entering the test section.

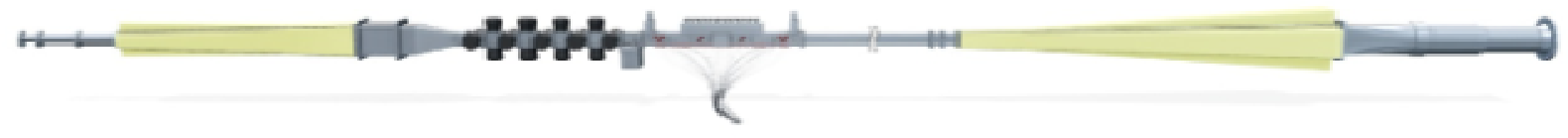

Figure 2. Artist's rendition of Grazing Flow Impedance Tube (GFIT).

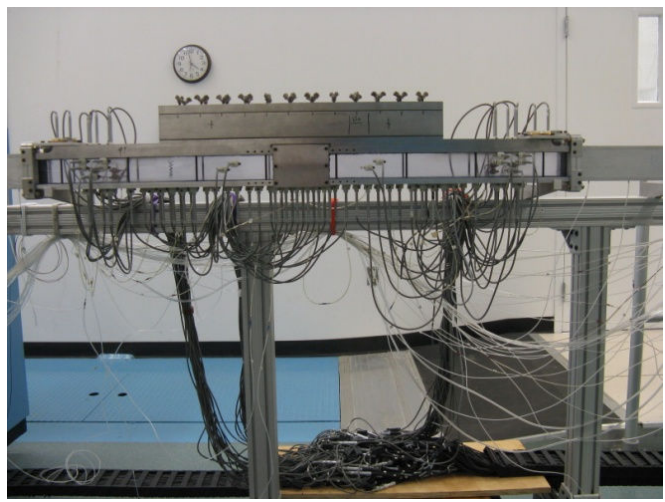

Figure 3. Photograph depicting GFIT test section with microphone instrumentation. 
Table 1. Characteristics and capabilities of the Grazing Flow Impedance Tube

\begin{tabular}{|l|l|}
\hline Test Section Dimensions & 2 in. $\times 2.5$ in. $\times 50$ in. $(\mathrm{w} \mathrm{x} \mathrm{h} \mathrm{x} 1)$ \\
\hline Operating Mach & 0.0 to 0.6 \\
\hline Operating Pressure & Ambient \\
\hline Operating Temperature & Ambient \\
\hline Maximum SPL & $160 \mathrm{~dB}$ \\
\hline Operating frequency range & 0.4 to $3.0 \mathrm{kHz}$ \\
\hline
\end{tabular}

\section{Data Acquisition}

The acoustic measurement system consists of Brüel and Kjær Type 4938 microphones, Type 2670 Preamplifiers, and Type 2690 Nexus Conditioning Amplifiers, combined with multiple National Instruments PXI-4496 Analog-to-Digital converter to acquire the data. A reference microphone is used to set the desired sound pressure level and the phase of each microphone in the acoustic array is referenced to that microphone. Data from all 95 microphones are collected in two acquisition passes (the reference microphone is sampled twice) of 48 channels per pass using a Cytec TXAR-128 Bank Switch. A PC using customized LabView data analysis software performs the spectral analysis and computes sound magnitude and phase at each microphone location. This process takes approximately two minutes per frequency.

The aerodynamic measurement system consists of varying instrumentation to measure pressure and temperature. Tunnel and traverse pressures are measured using a Ruska Series Type 6200, Mensor Model 14000, and Mensor Model 2102 equipment. Static pressures along the length of the test rig are measured using Pressure Systems Netscanner 9116 16-port Pressure Measurement Modules. Tunnel and traverse temperatures are measured using National Instruments NI-4351 Thermocouple/Voltage Logger equipment.

\section{Definition of Uncertainty Metrics}

The purpose of the uncertainty analysis is to define specification limits for the variation of acoustic impedance about a target value. This is particularly challenging when working with the acoustic impedance, as this parameter generally varies with frequency, mean flow speed, and source sound pressure level. For this reason, uncertainty metrics are defined for each liner at each Mach number over the operating range of discrete frequencies.

In a general experiment, an unbiased measurement repeated $N$ times on a statistically stable, parent population, will have random fluctuations about the mean of $N$ samples. For a Gaussian (also known as normal or unimodal), distribution, the sample size mean derived from the parent population approaches the mean of the parent population (or "true mean"). Also, the following assumptions are made regarding the measurement space: (1) the parent population is Gaussian distributed, (2) any sampling distribution (sample size from the parent population) is symmetric and unimodal, and (3) the "true value" of a measurement is assumed to be the mean value calculated from repeated measurements. In practice, due to budget and time constraints, it is impractical to conduct a large number of repeat trials. Hence, results provided herein are based on "small sample statistics" $(N=11)$.

In this study, the metric for uncertainty is chosen to be the $95 \%$ confidence interval (CI). These intervals are calculated from the statistics generated by repeated aerodynamic measurements and educed impedance at each frequency. There are two types of $95 \%$ CI used in this study. The first provides a $95 \%$ confidence limit for determining the "true mean" values of the normalized resistance and reactance. This is used to indicate the user can be $95 \%$ confident that the true mean (i.e., the mean value that would be calculated if an infinite number of tests could be conducted) will fall between the upper and lower limits of the confidence interval. This terminology will be labeled as $\mathrm{CI}_{\text {mean }}$. The upper and lower limits are computed by using Eq. 1, where: $\bar{x}$ is the mean value averaged over $N$ trials, $t^{*}$ is the critical value for the t-distribution with $N-1$ degrees of freedom (i.e., $t(N-1)$ ), $s$ is the estimated standard deviation of a small number of trials $(N<32)$, and $N$ is the number of trials. In this experiment $N=11$, and the critical value $t^{*}$ for $95 \%$ confidence interval for 10 degrees of freedom is $2.23 .^{2}$

$$
C I_{\text {true mean }}=\bar{x} \pm t^{*} \frac{s}{\sqrt{N}}
$$


The second provides the corresponding 95\% confidence limit related to the uncertainty of the "next measurement." This is used to indicate the user can be $95 \%$ confident that the next measurement (from the educed impedance spectrum) will fall between the upper and lower limits. This terminology will be labeled as $\mathrm{CI}_{\text {next }}$. The upper and lower limits for computing $\mathrm{CI}_{\text {next }}$ are computed using Eq. 2.

$$
C I_{\text {next }}=\bar{x} \pm t^{*} S
$$

The spread between the upper and lower limits of $\mathrm{CI}_{\text {next }}$ is larger than that for $\mathrm{CI}_{\text {mean }}$. These definitions will be discussed more in detail in the Results and Discussion section of this paper.

\section{Description of Experiment}

An illustration of a multi-purpose liner sample for GFIT testing is shown in Fig. 4. The liner sample consists of a facesheet that serves as the upper wall of the test section, a core liner, and a backplate. Two singlelayer liner samples used in this investigation are outlined in Table 2. The characteristics include: liner type (nonlinear and linear); upper layer characterized by either: (1) facesheet with circular hole diameter, thickness, and percent open area or (2) wire mesh; liner treated region.

The current study is restricted to one SPL $(130 \mathrm{~dB})$ and three Mach numbers $(0.0,0.3,0.5)$, over a range of frequencies from 0.4 to $3 \mathrm{kHz}$. Each test configuration (liner, Mach, SPL, frequency) is tested eleven times such that small sample statistics ${ }^{2}$ can be used to evaluate uncertainty in the results.

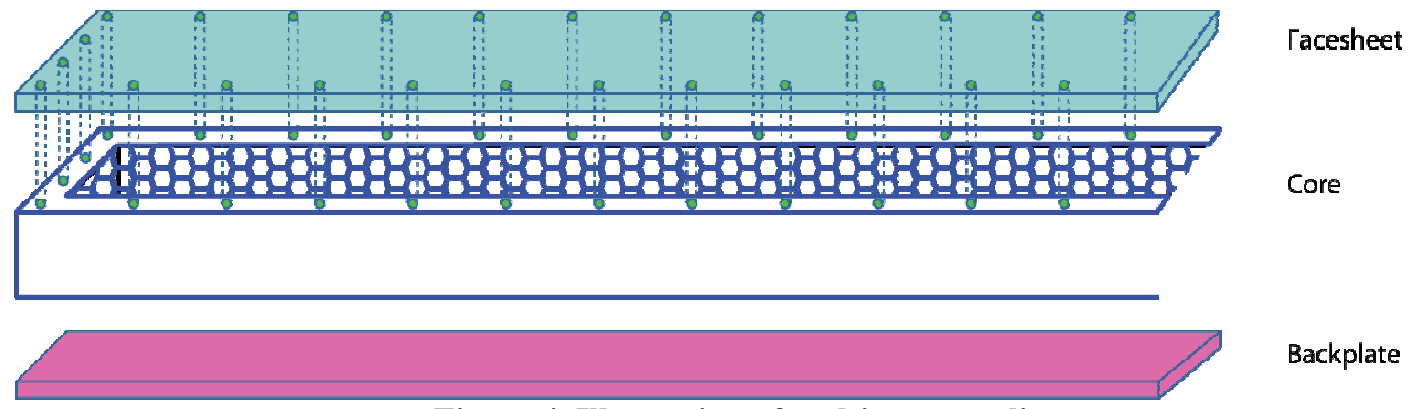

Figure 4. Illustration of multi-purpose liner.

Table 2. Liner Sample Geometry

\begin{tabular}{|c|c|c|c|c|c|c|c|}
\hline $\begin{array}{l}\text { Liner } \\
\text { Sample }\end{array}$ & $\begin{array}{l}\text { Liner } \\
\text { Type }\end{array}$ & $\begin{array}{l}\text { Hole } \\
\text { Diameter } \\
\text { (in) }\end{array}$ & $\begin{array}{l}\text { Facesheet } \\
\text { Thickness } \\
\text { (in) }\end{array}$ & $\begin{array}{l}\text { POA } \\
(\%)\end{array}$ & $\begin{array}{l}\text { Cavity } \\
\text { Depth } \\
\text { (in) }\end{array}$ & $\begin{array}{c}\text { Wire Mesh (Sefar) } \\
\text { (MKS Rayls) }\end{array}$ & $\begin{array}{l}\text { Treated Region } \\
\text { Coordinates } \\
\text { (in) }\end{array}$ \\
\hline Liner 1 & Nonlinear & 0.039 & 0.025 & 8.7 & 1.5 & & $8.3 \leq \mathrm{z} \leq 24.056$ \\
\hline Liner 2 & Linear & & & & 2.0 & 570 & $8.25 \leq \mathrm{z} \leq 31.75$ \\
\hline
\end{tabular}

\section{Acoustic Impedance Eduction Process}

The key parameter used to assess the acoustic performance of a liner is acoustic impedance, the ratio of acoustic pressure to the normal component of acoustic particle velocity. The liner acoustic impedance is determined by an iterative process, in which multiple liner impedances are used as input to a duct acoustics propagation code based on the convected Helmholtz equation. The one that provides the acoustic pressure profile that most closely matches the measured acoustic pressure profile is assumed to be the impedance of the liner. ${ }^{3,4}$ 
The NASA Langley acoustic impedance eduction methodology $y^{5,6}$ is described briefly in this section. Fig. 5 provides a rendition of the GFIT defining the physical domain used for educing acoustic impedance. The acoustic liner forms part of the upper wall over the range $L_{1} \leq z \leq L_{2}$ and the remaining domain contains rigid walls. Microphones on the lower wall measure acoustic pressure over the range $0 \leq z \leq L$. The source and exit planes are located at $z=0$ and $z=L$, respectively.

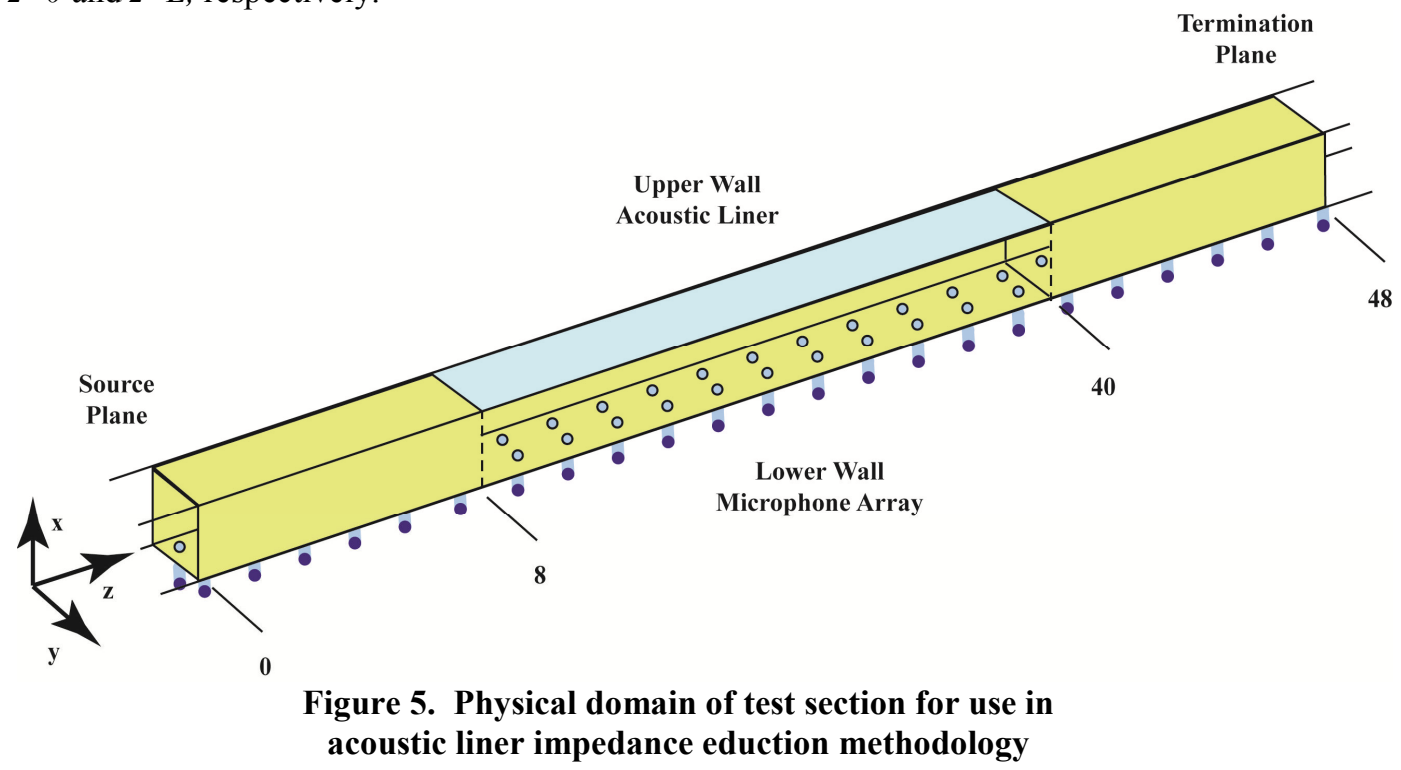

The impedance eduction technique begins by assuming the acoustic liner is unknown and is defined by a uniform, normalized impedance, $\zeta$. The acoustic pressure (magnitude and phase) is assumed to be known, measured by the microphone array on the lower wall. A uniform flowfield is also assumed. A major advantage of this flow model is that the differential equations that govern the acoustic field can be combined into a single differential equation involving one variable. The source is assumed to be a single tone at an excitation frequency such that no higher-order spanwise modes propagate, which reduces the computational domain to the $(\mathrm{x}, \mathrm{z})$ - plane.

The acoustic pressure field, $p$, propagating through the flow duct under the defined conditions satisfies the convected Helmholtz equation:

$$
\left(1-M_{0}\right) \frac{\partial^{2} p}{\partial z^{2}}+\frac{\partial^{2} p}{\partial x^{2}}-2 i k M_{0} \frac{\partial p}{\partial z}+k^{2} p=0
$$

where $k=\omega / c$ is the freespace wavenumber and $M_{0}$ is the mean flow Mach number. The angular frequency, $\omega$, and the excitation frequency, $f$, are related by the following expression: $\omega=2 \pi f$.

For the upper wall, the Ingard-Myers boundary condition $^{3,4}$ is:

$$
-\frac{\partial p}{\partial x}=i k\left(\frac{p}{\zeta}\right)+2 M_{0} \frac{\partial}{\partial z}\left(\frac{p}{\zeta}\right)+\frac{M_{0}^{2}}{i k} \frac{\partial^{2}}{\partial z^{2}}\left(\frac{p}{\zeta}\right)
$$

where $\zeta$ represents the unknown normalized acoustic impedance of the upper wall. The admittance $(1 / \zeta)$ of the liner is zero for the hard wall sections.

The boundary condition of the hard lower wall is: 


$$
\frac{\partial p}{\partial x}=0
$$

The source plane microphone is located upstream of the liner at $\mathrm{z}=0$. This microphone is flush-mounted in the hard wall section opposite (lower wall) the test liner.

The microphone used to measure the acoustic pressure profile, $p_{s}(x)$ in the source plane (Fig. 5, designated by $z=0$ ) is used to define the source plane boundary condition:

$$
p(0, x)=p_{s}(x)
$$

The excitation frequency is kept below the cut-on of higher-order modes in the hard wall sections. The acoustic pressure at the source plane microphone provides, therefore, a measurement of the sound source pressure profile, $p(0,0)=p_{s}(x)$. Similarly, there is a lower wall flush-mounted microphone at the exit plane of the acoustic test liner (Fig. 5, designated by $L$ ) that is used to determine the exit plane acoustic pressure boundary condition, $p_{e}(x)$ :

$$
p(L, x)=p_{e}(x)
$$

The impedance eduction technique is used to solve the boundary value problem defined by Eqs. 3-7 using a finite element method (FEM) with cubic Hermite polonomial functions. An initial value of an unknown acoustic impedance, $\zeta$, is educed by an iterative process that converges on an optimum impedance that reproduces the acoustic pressure field by the flush-mounted microphones on the hard lower wall (see Fig. 5). This is achieved by minimizing the objective function:

$$
F(\zeta)=\sum_{I=1}^{n w a l l}\left\{\left.p\left(z_{I}, 0\right)\right|_{\text {FEM }}-\left.p\left(z_{I}, 0\right)\right|_{\text {Meas }}\right\}\left\{\left.p^{*}\left(z_{I}, 0\right)\right|_{F E M}-\left.p^{*}\left(z_{I}, 0\right)\right|_{\text {Meas }}\right\}
$$

where: nwall is the number of flush-mounted microphones on the hard lower wall; $\left.p\left(z_{I}, 0\right)\right|_{\text {FEM }}$ is the acoustic pressure predicted at the microphone using the finite element method at $z=z_{I} ;\left.\quad p\left(z_{I}, 0\right)\right|_{\text {Meas }}$ is the acoustic pressure measured at the flush-mounted microphone located at $z=z_{I}$; and the superscript * denotes the complex conjugate of the acoustic pressure. The unknown acoustic impedance, $\zeta$ is determined by finding the impedance that causes this objective function to be minimized. Minimizing the objective function is achieved using Stewart's adaptation of the Davidon-Fletcher-Powell (SDFP) optimization algorithm. ${ }^{7}$ The optimization algorithm returns the normalized resistance, $\theta$, and the normalized reactance, $\chi$, of the test liner acoustic impedance, $\zeta=\theta+i \chi$. The SDFP algorithm requires only an initial starting value for $\zeta$. For the results presented in this abstract, the initial starting values are (1) $\zeta=0.5+2.0 i$; (2) $\zeta=0.5-2.0 i$; (3) $\zeta=2.0+2.0 i$; and (4) $\zeta=2.0-2.0 i$.

\section{Results}

The uncertainty analysis methodology used in this study is similar to that used in the previous investigation of Jones, et al., ${ }^{8}$ which is based on Coleman and Steele's ${ }^{2}$ work. The purpose of this investigation is three-fold: (1) to conduct uncertainty analysis with linear and nonlinear liners, to determine the effects of linearity on the $95 \%$ confidence intervals, and (2) to establish a streamlined methodology for determining the uncertainty associated with acoustic impedance measurements at NASA LaRC.

Figures 6 through 12 provide representative normalized resistance, $\theta$, and reactance, $\chi$, spectra from the uncertainty analysis based on 11 trials with each liner at each condition of interest (liner, Mach number, frequency). Small sample statistics based on Student's t-distribution ${ }^{2}$ are used to process the results, where it is assumed that the input (e.g. static temperature, static pressure, Mach number) and output (acoustic impedance) parameters used in the impedance eduction process are normally distributed (i.e. Gaussian). Every figure is plotted on the same scale so the 
reader can easily see the changes in impedance spectra for each flow regime. The normalized resistance and reactance spectra over the frequency range are provided on the same plot. The diamond and square symbols represent the mean acoustic resistance and reactance, respectively. The upper and lower $95 \%$ confidence levels are represented as error bars for each frequency. Note that the frequencies where the error bars are not visible indicate the confidence intervals are not visibly discernible in the $2^{\text {nd }}$ or $3^{\text {rd }}$ decimal place. At $\mathrm{M}=0.0$, most of the error bars are not easily discernible, indicating very good repeatability. At $M=0.3$ and $M=0.5$, the majority of the scatter occurs at the lower frequencies, and becomes largest in the vicinity of anti-resonance (where the magnitude of the resistance approaches a large value).

As the flow Mach number is increased from 0.0 to 0.5 , the normalized resistance is observed to increase. The trend is also observed to change. At $\mathrm{M}=0.0$, the resistance is essentially frequency independent. However, at Mach numbers of 0.3 and 0.5 the resistance decreases with increasing frequency. These flow results are not predicted well by most impedance models, and are believed to be due to limitations in the impedance eduction process caused by the assumption of uniform flow. ${ }^{9}$ This is expected to be the focus of a future investigation. Nevertheless, the current results demonstrate that the current impedance eduction process is repeatable, as indicated by the small confidence intervals.

Figure 9 provides the same normalized impedance spectrum for $\mathrm{M}=0.5$, with one exception. This figure shows the $95 \% \mathrm{CI}$ for the next measurement at each frequency. This confidence interval is significantly larger at each frequency than the corresponding $95 \% \mathrm{CI}$ for the true mean. This indicates that it is much more difficult to predict the accuracy of the next occurrence than to predict the true mean from the previously acquired data. Similar results were attained for $\mathrm{M}=0.0$ and 0.3 at each frequency, however, the confidence intervals were very small, making any error bars not easily discernible; therefore these results are not presented in this paper.

Figures 10 and 11 represent the normalized resistance and reactance of Liner 2 (linear liner) over the frequency range for flow Mach numbers of 0.3 and 0.5 . The error bars represent $95 \% \mathrm{CI}$ of the true mean at each frequency. The $\mathrm{M}=0.0$ are not shown, as the scatter in impedance was too small to visualize on this scale. Like Liner 1, with flow the majority of scatter occurs at the lower frequencies, becoming largest in the vicinity of anti-resonance.

Figure 12 provides the $95 \% \mathrm{CI}$ at the next measurement at each frequency for the $\mathrm{M}=0.5$ data. As shown in Fig. 9, these confidence intervals are noticeably larger than those for the true mean. However, the CI limits are much smaller for Liner 2 (linear) than for Liner 1 (nonlinear).

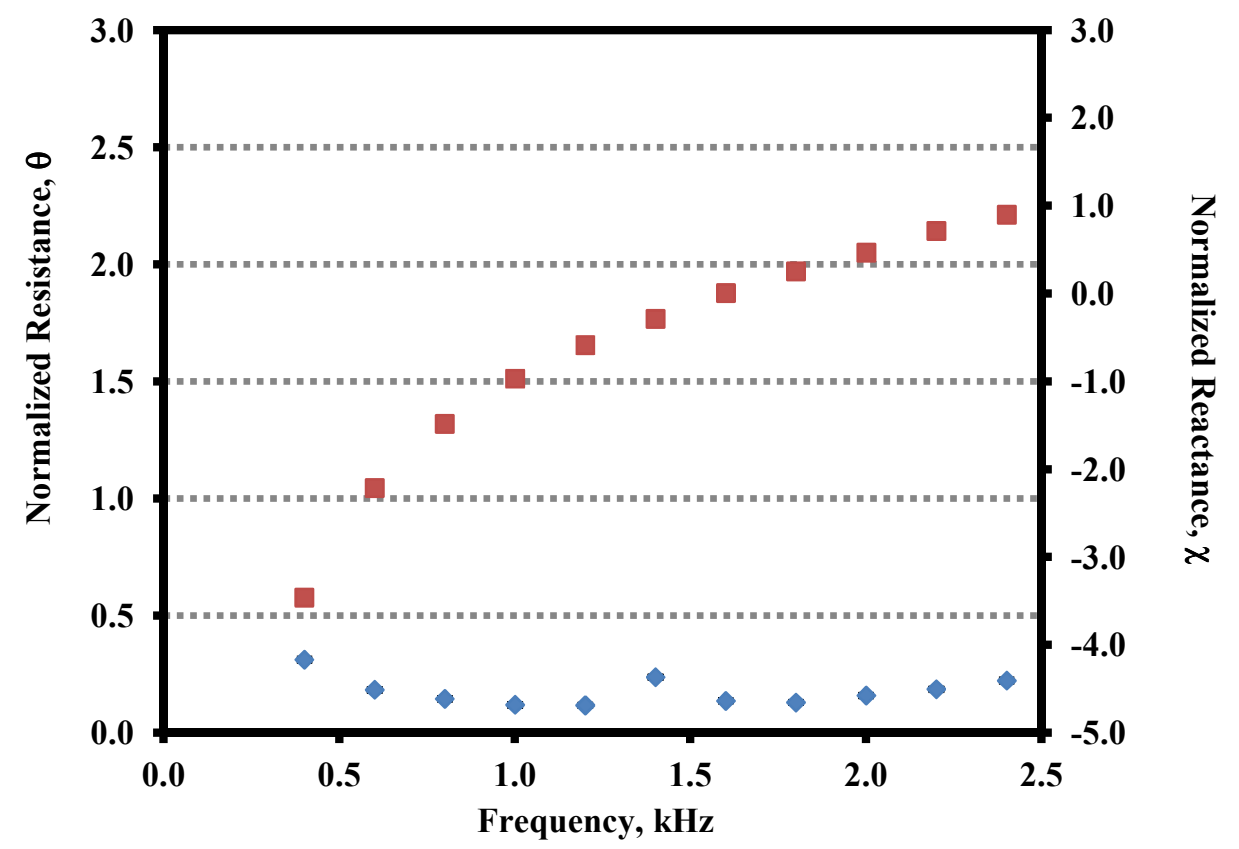

Figure 6. Normalized acoustic resistance (diamonds) and reactance (squares) versus frequency at $\mathbf{M = 0 . 0}$. Liner 1; Error bars denote $\mathbf{C I}_{\text {mean }}$. 


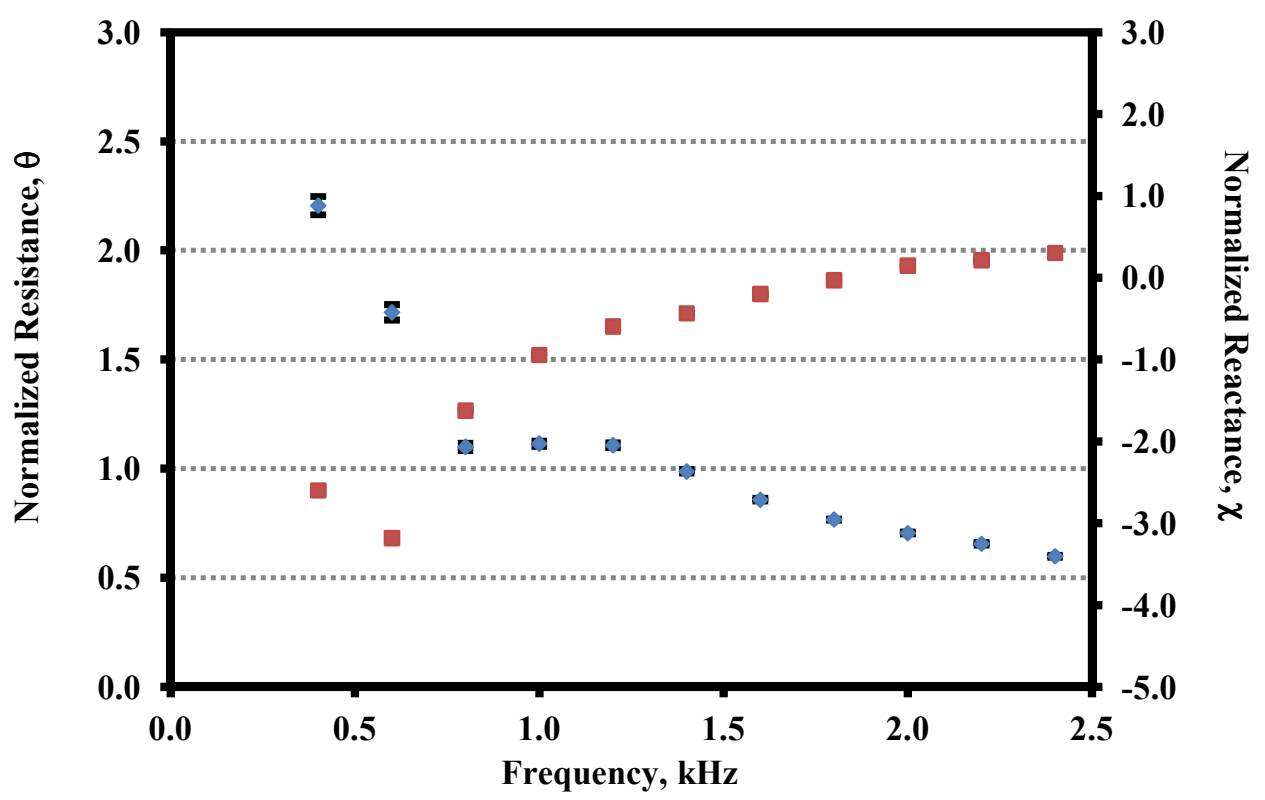

Figure 7. Normalized acoustic resistance (diamonds) and reactance (squares) versus frequency at $\mathbf{M}=\mathbf{0 . 3}$. Liner 1; Error bars denote $\mathbf{C I}_{\text {mean }}$.

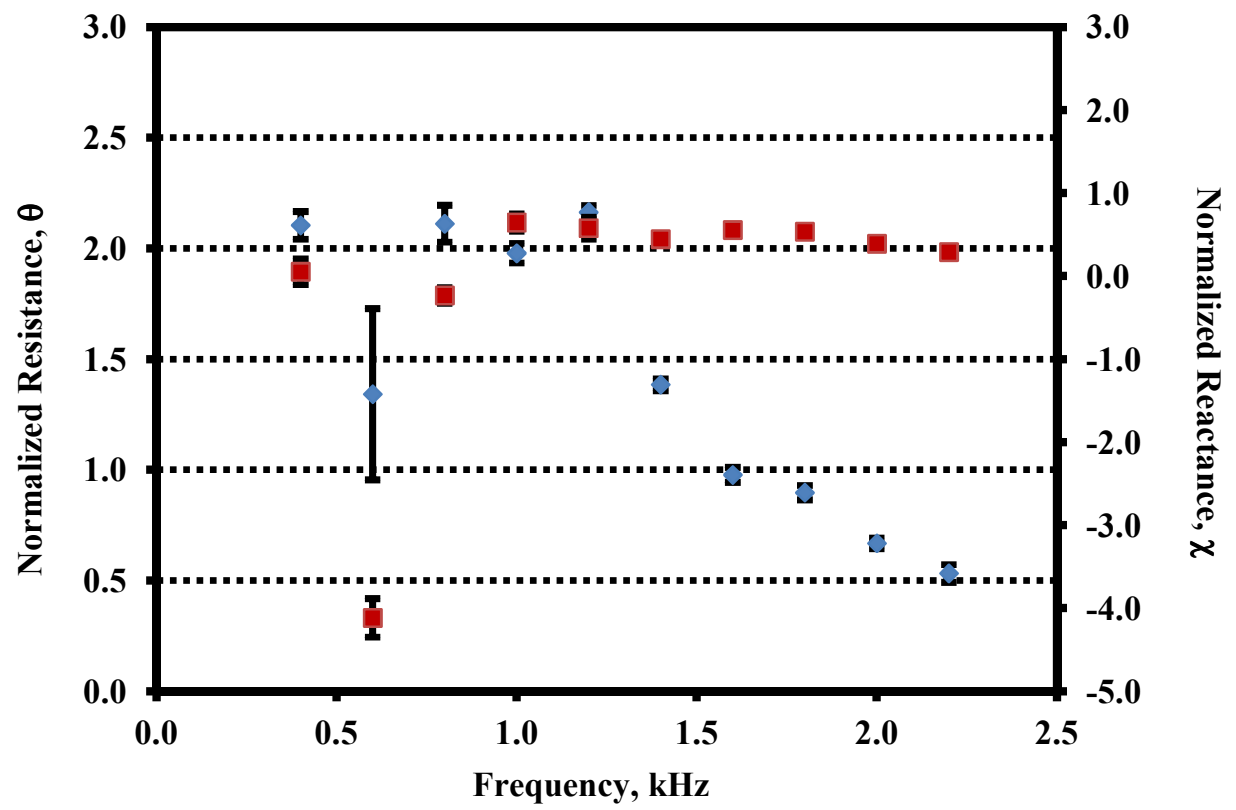

Figure 8. Normalized acoustic resistance (diamonds) and reactance (squares) versus frequency at $M=0.5$. Liner 1; Error bars denote $\mathbf{C I}_{\text {mean }}$. 


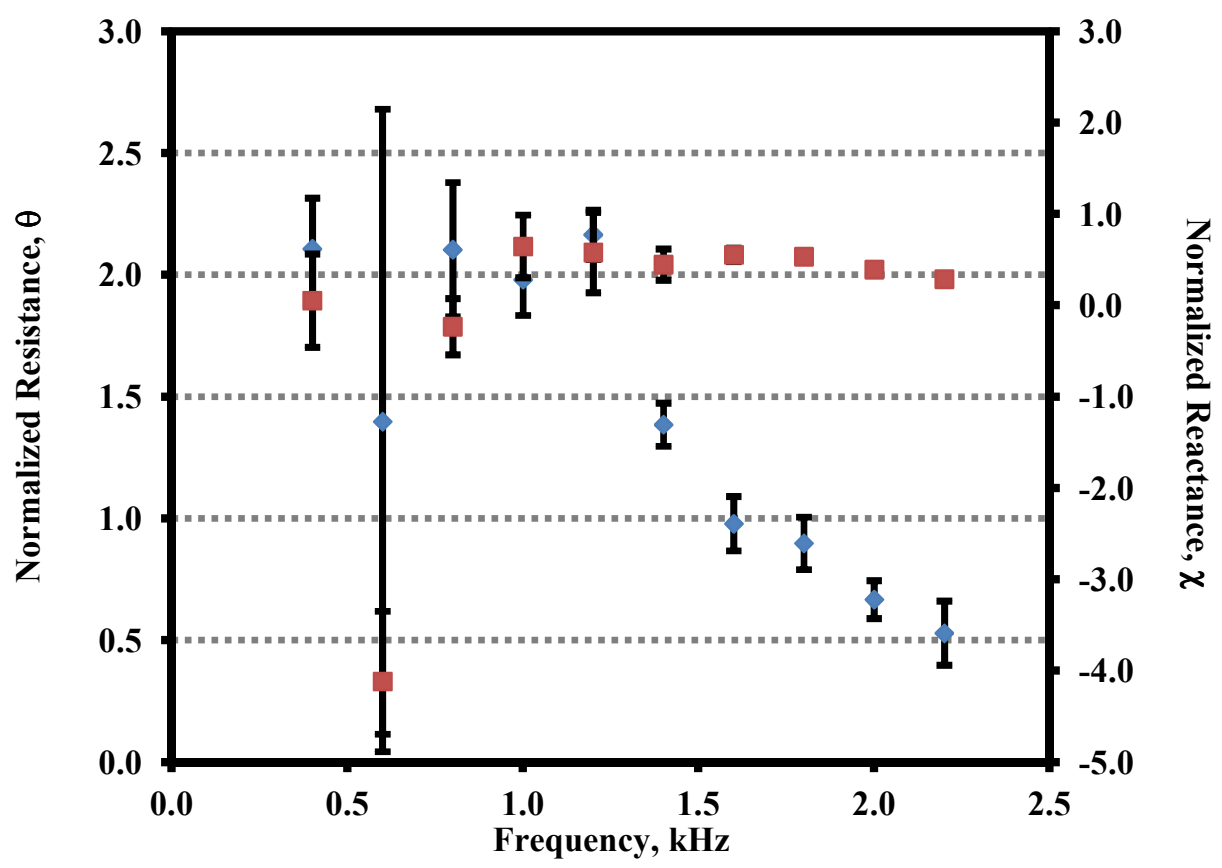

Figure 9. Normalized acoustic resistance (diamonds) and reactance (squares) versus frequency at $M=0.5$. Liner 1; Error bars denote $\mathbf{C I}_{\text {next }}$.

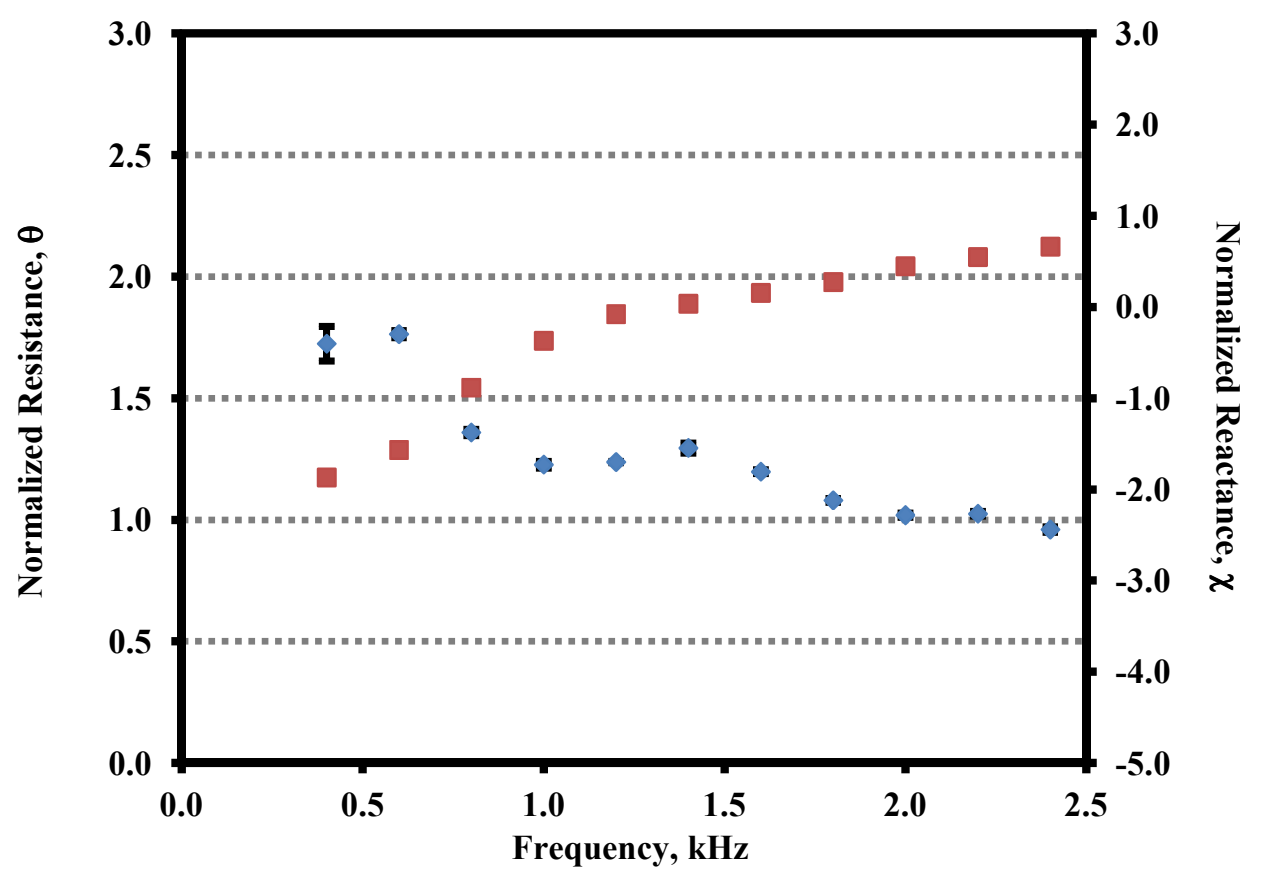

Figure 10. Normalized acoustic resistance (diamonds) and reactance (squares) versus frequency at $M=0.3$. Liner 2; Error bars denote $\mathbf{C I}_{\text {mean }}$. 


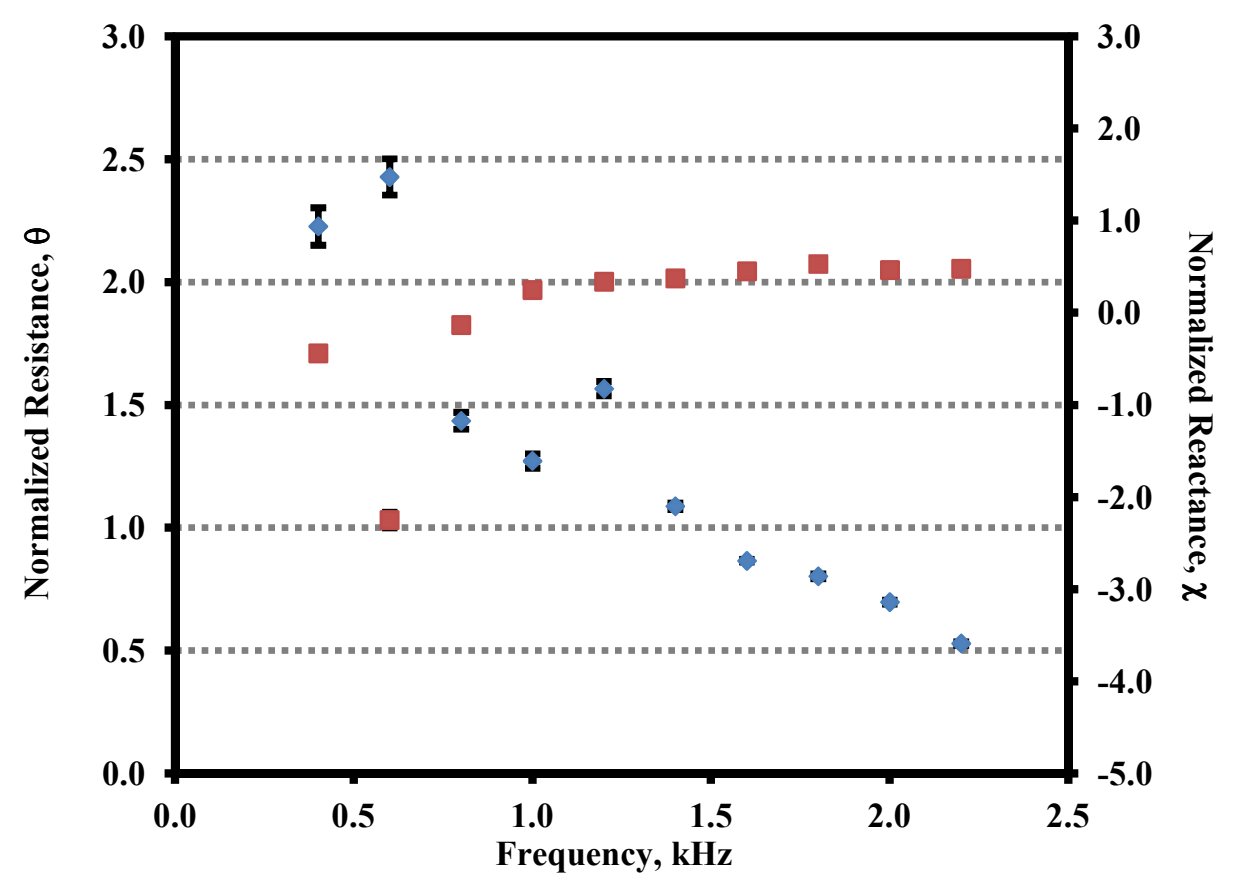

Figure 11. Normalized acoustic resistance (diamonds) and reactance (squares) versus frequency at $\mathrm{M}=\mathbf{0 . 5}$. Liner 2; Error bars denote $\mathbf{C I}_{\text {mean }}$.

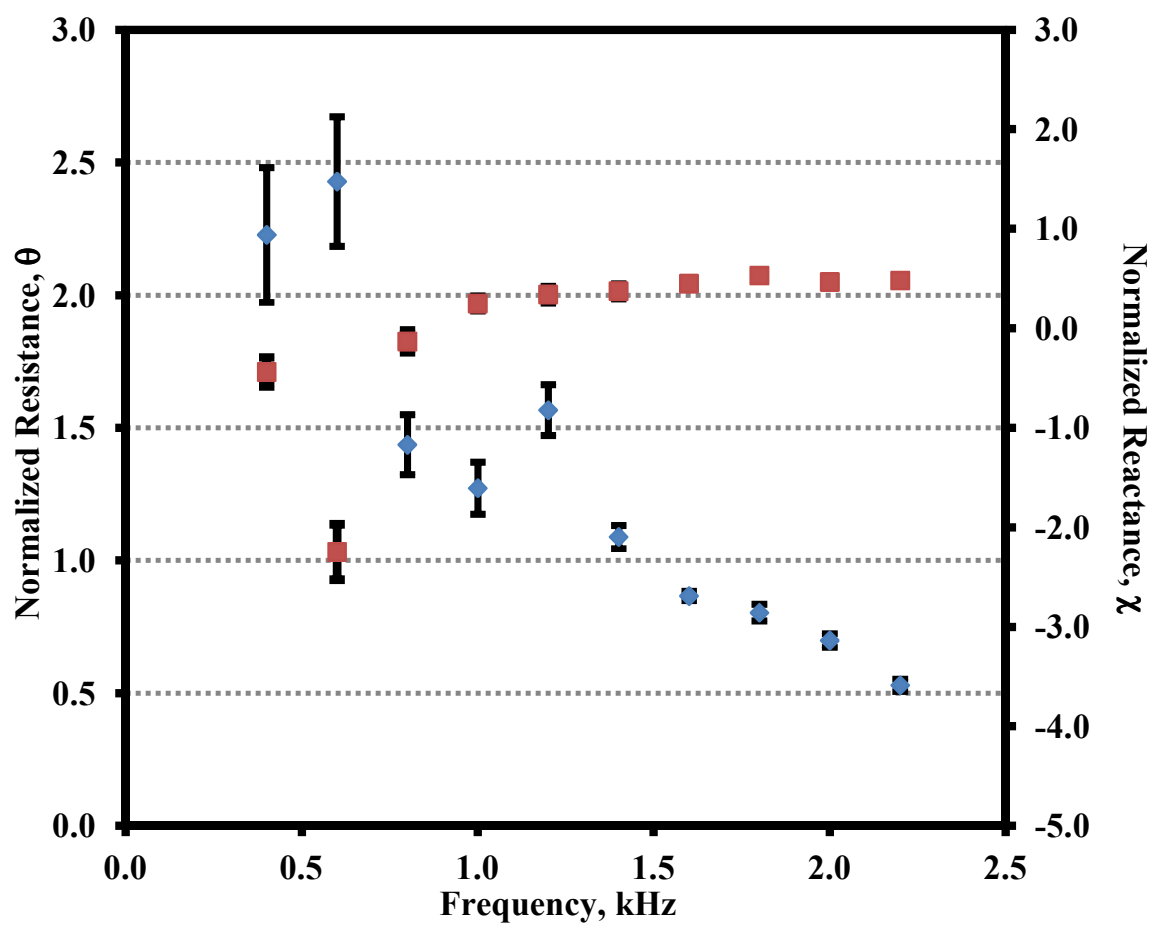

Figure 12. Normalized acoustic resistance (diamonds) and reactance (squares) versus frequency at $M=0.5$. Liner 2; Error bars denote $\mathbf{C I}_{\text {next }}$. 
A sensitivity analysis of the aerodynamic parameters used in the impedance eduction process was also conducted. A Monte-Carlo approach similar to that performed by Nark, ${ }^{10}$ et al. was employed to investigate the aerodynamic parameters of average Mach number, $\mathrm{M}_{\text {ave }}$, static pressure, $\mathrm{P}_{\mathrm{s}}$, and static temperature, $\mathrm{T}_{\mathrm{s}}$. The purpose of this study is to determine the significance of each aerodynamic parameter to the acoustic attenuation over the axial length of the liner sample using a duct acoustic propagation prediction code based on the convected Helmholtz equation (CHQ3D). ${ }^{10}$ For desired inputs $\left(\mathrm{M}_{\text {ave }}, f, \mathrm{P}_{\mathrm{s}}, \mathrm{T}_{\mathrm{s}}, \theta, \chi\right)$, CHQ3D computes the attenuation over the axial length of the test section.

The scatter in $\mathrm{M}_{\text {ave }}$ and $\mathrm{P}_{\mathrm{s}}$ were found to be virtually imperceptible. Thus, only the $\mathrm{T}_{\mathrm{s}}$ results will be presented. Table 3 outlines the input parameters to CHQ3D. The upper and lower limits on $\mathrm{T}_{\mathrm{s}}$ were derived using 95\% CI True Mean over 11 trials. Three frequencies $(1.0,1.6$, and $2.0 \mathrm{kHz})$ are used to demonstrate the results of the MonteCarlo process. Inputs for $T_{s}$ were randomized 100 times over the $95 \%$ confidence interval. Values for resistance and reactance are the mean values.

The acoustic propagation code CHQ3D, was run for each $\mathrm{T}_{\mathrm{s}}$. Figure 13 is a representative plot of SPL over the axial length in the computational domain. There are three sections to this curve: (A) the curve for $L<8$ inches is the portion of the test section that is upstream of the acoustically treated section; (B) steep decay over the acoustically treated section; (C) hardwall section downstream of the liner.

For each CHQ3D prediction (one per $\mathrm{T}_{\mathrm{s}}$ ), an attenuation slope over the length of the liner was determined. The range of slopes (maximum minus minimum) is shown in Table 4 for each liner, at an average Mach number of 0.403 , and at frequencies of 1.0, 1.6, and $2.0 \mathrm{kHz}$. Note that the differences between the minimum and maximum slopes are very small. Therefore, the Monte-Carlo process demonstrates that the static temperature contribution to the impedance eduction uncertainty can safely be ignored.

Table 3. CHQ3D input parameters.

\begin{tabular}{|c|c|c|c|c|c|c|}
\hline Liner & $\mathrm{M}_{\mathrm{ave}}$ & $\begin{array}{c}\text { Freq } \\
(\mathrm{kHz})\end{array}$ & $\begin{array}{c}\mathrm{P}_{\mathrm{s}} \\
\text { (psia) }\end{array}$ & $\begin{array}{c}\mathrm{T}_{s}\left({ }^{\circ} \mathrm{F}\right) \\
{[\mathrm{min}, \max ]}\end{array}$ & Resistance, $\theta$ & Reactance, $\chi$ \\
\hline \multirow{3}{*}{ Liner 1} & \multirow{3}{*}{0.403} & 1.0 & \multirow{3}{*}{14.732} & \multirow{3}{*}[49.5,50.7]{} & 1.978 & 0.645 \\
\hline & & 1.6 & & & 0.977 & 0.554 \\
\hline & & 2.0 & & & 0.668 & 0.392 \\
\hline \multirow{3}{*}{ Liner 2} & \multirow{3}{*}{0.403} & 1.0 & \multirow{3}{*}{14.668} & \multirow{3}{*}[50.6,52.0]{} & 1.272 & 0.249 \\
\hline & & 1.6 & & & 0.866 & 0.450 \\
\hline & & 2.0 & & & 0.698 & 0.464 \\
\hline
\end{tabular}

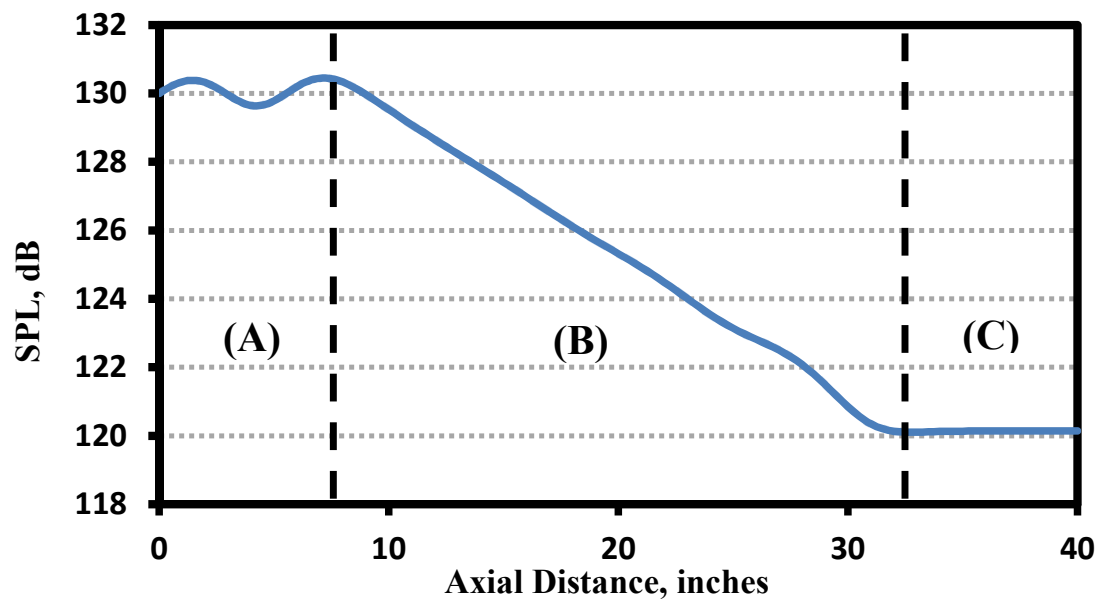

Figure 13. SPL attenuation vs. axial distance. Liner 2; Frequency: $1.6 \mathrm{kHz} ; \mathrm{M}_{\mathrm{ave}}=0.403$. 
Table 4. Predicted minimum and maximum attenuation per axial distance; $M_{a v e}=0.403$.

\begin{tabular}{|c|c|c|c|c|c|}
\hline \multirow{2}{*}{ Liner } & $\begin{array}{c}\text { Freq } \\
(\mathrm{kHz})\end{array}$ & Resistance, $\theta$ & Reactance, $\chi$ & $\mathrm{dB} /$ in $(\max )$ & $\mathrm{dB} / \mathrm{in}(\min )$ \\
\hline \multirow{3}{*}{ Liner 1 } & 1.0 & 1.978 & 0.645 & 0.4240 & 0.4241 \\
\cline { 2 - 6 } & 1.6 & 0.977 & 0.554 & 0.6124 & 0.6129 \\
\cline { 2 - 6 } & 2.0 & 0.668 & 0.392 & 0.6403 & 0.6411 \\
\hline \multirow{3}{*}{ Liner 2 } & 1.0 & 1.272 & 0.249 & 0.7038 & 0.7038 \\
\cline { 2 - 6 } & 1.6 & 0.866 & 0.450 & 0.7075 & 0.7082 \\
\cline { 2 - 6 } & 2.0 & 0.698 & 0.464 & 0.5812 & 0.5820 \\
\hline
\end{tabular}

\section{Concluding Remarks}

A methodology to identify the measurement uncertainty of the impedance eduction process for data acquired in the GFIT has been demonstrated. This methodology was used to evaluate results acquired with two liners, one linear and the other nonlinear, with respect to source sound pressure level. The results were computed based on $95 \%$ confidence intervals.

The confidence intervals increase (i.e., measured uncertainty increases) with Mach number. Also, the effects of measured uncertainty are larger for the nonlinear liner. For both liners, the confidence intervals are also larger at frequencies near anti-resonance.

The uncertainty analysis process described in this study will continue to be used to monitor the effects of measured uncertainty for tests conducted in the GFIT. This approach will also be applied for tests conducted in other NASA test rigs, e.g. the Curved Duct Test Rig.

\section{Acknowledgments}

The authors would like to express appreciation to Brian Howerton and Carol Harrison of NASA Langley Research Center, as well as Jerry Lyle of Northrop Grumman Corporation for their contributions to this study. This work was funded by the Subsonic Fixed Wing Project of NASA's Fundamental Aeronautics Program.

\section{References}

${ }^{1}$ Jones, M.G., Parrott, T.L., and Watson, W.R.: "Uncertainty and Sensitivity Analysis of a Two-Parameter Impedance Prediction Model," AIAA-2008-2928, June 2008.

${ }^{2}$ Coleman, H.W. and Steele, W.G.: Experimentation and Uncertainty Analysis for Engineers, $2^{\text {nd }}$ Edition, John Wiles \& Sons, Inc., 1999.

${ }^{3}$ Ingard, K.U.: "Influence of Fluid Motion Past a Plane Boundary on Sound Reflection, Absorption, and Transmission," Journal of Acoustic Society of America, vol. 31 no.7, pp. 1035-1036, 1959.

${ }^{4}$ Myers, M.K.: "On the Acoustic Boundary Condition in the Presence of Flow," Journal of Sound and Vibration, vol. 71, no. 3, pp. 429-434, 1980.

${ }^{5}$ Watson W. R., Jones, M. G., Tanner, S.E., Parrot, T.L.: “A finite element propagation model for extracting normal incidence impedance in nonprogressive acoustic wave fields," Journal of Computational Physics, Vol. 125, 1996.

${ }^{6}$ Watson W. R., Jones, M. G.: "Explanation of Anomalous Behavior Observed in Impedance Eduction Techniques Using Measured Data," AIAA 2010-3766, 2010.

${ }^{7}$ Stewart, G.W., III: "A Modification of Davidson's Minimization Method to Accept Difference Approximations of Derivatives," Journal of ACM, vol. 14, No. 1, 1967, pp 72-83.

${ }^{8}$ Jones, M.G., Parrott, T.L., and Watson, W.R.: "Uncertainty and Sensitivity Analysis of a Two-Parameter Impedance Prediction Model,” AIAA-2008-2928, June 2008.

${ }^{9}$ Jones, Michael G., and Watson, Willie R.: "On the Use of Experimental Methods to Improve Confidence in Educed Impedance," AIAA-2011-2865, June 2011.

${ }^{10}$ Nark, Douglas M., Watson, Willie R., and Jones, Michael G.: "Uncertainty and Sensitivity Analyses of Duct Propagation Models," AIAA-2008-2832, June 2008. 